\title{
Perspectiva ético-política de las pedagogías críticas*
}

\section{Ethical and political perspectives of pedagogy criticisms}

\author{
Piedad Ortega Valencia \\ Diana Peñuela Contreras**
}

Resumen:

En el presente artículo se problematizan varios aspectos abordados por el grupo de Investigación "Sentidos y configuraciones de las pedagogías críticas en Colombia". Un primer asunto se inscribe en la discusión de las pedagogías críticas como un campo emergente en constitución, con un recorrido histórico, pedagógico y político específico para nuestro país; en segundo lugar, se exponen algunas consideraciones sobre las pedagogías críticas en su compresión como discursos y prácticas de resistencia; y finalmente, pretende realizar algunos planteamientos en relación con su componente ético-político.

\section{Palabras claves:}

Pedagogía, sujetos, campo, contexto, discursos.

\section{Abstract:}

This paper points out some problems with certain aspects studied by the research group Senses and configurations of the critical pedagogies in Colombia. First, the discussion is about critical pedagogies as an emerging field developing with a historical, political and pedagogical background specific to our country. Second, the paper presents some reflections about the critical pedagogies in its comprehension as a practice and discourse of resistance. Finally, it tries to risk some approaches related to its ethical and political components.

Keywords:

Pedagogy, subjects, field, context, discourses

Artículo recibido el 30 de julio de 2007 y aprobado el 31 de octubre de 2007

* Este artículo se presenta como avance del proyecto de investigación "Sentidos y configuraciones de las pedagogías críticas en Colombia”, en el cual integran el grupo de investigación: Lola Cendales, Diana Peñuela, Francy González, Luis Hurtado y Piedad Ortega. Proyecto financiado por el Centro de Investigaciones de la Universidad Pedagógica Nacional, CIUP.

** Profesoras de la Universidad Pedagógica Nacional. piedadortegava@yahoo.es, dianami29@gmail.com 


\section{Las pedagogías críticas como un campo emergente en constitución.}

No te salves

No te quedes inmóvil al borde del camino no congeles el júbilo no quieras con desgana no te salves ahora ni nunca.

Mario Benedetti

La primera consideración que deseamos exponer es que no proponemos el Campo de las Pedagogías Críticas funcionando de forma aislada en el mapa educativo, sino que lo evidenciamos funcionando de manera concomitante ${ }^{1}$ con otros campos que han sido delimitados históricamente por discursos de intelectuales, los cuales han adquirido, por lo menos para Colombia, el estatuto de paradigmáticos, éstos son: el Campo Intelectual de la Educación, propuesto por Mario Díaz y el Campo Conceptual de la Pedagogía, planteado por Olga Lucía Zuluaga y Alberto Echeverri.

La particularidad del campo de las pedagogías críticas en nuestro país se da quizás en la relación más cercana que se atribuye a la pedagogía y a la producción de saber pedagógico con el contexto social, político, económico y cultural, que condiciona pero no determina históricamente, como diría Freire, la educación y el devenir del maestro como sujeto pedagógico, social y político. Así, el énfasis se inscribe en la relación pedagogía-política, por la lectura crítica que se le otorga a las relaciones de poder existentes y constituyentes del ámbito educativo entre los sujetos, discursos, instituciones, prácticas, leyes, normatividad y también procesos de movilización y resistencia que han co-existido históricamente en el mismo.

1 Que actúa, acompaña o colabora en el mismo sentido que otra cosa. Se trata entonces de acciones no excluyentes con puntos de diferenciación y aspectos específicos que las dotan de particularidades específicas.
No se trata de politizar la pedagogía ni tampoco de despedagogizar la práctica pedagógica misma; nuestra intención es reconceptualizar históricamente hechos, movilizaciones y acciones de resistencia desde el aula, pero también externas a ésta, que aunque se pueden incluir dentro del campo educativo general, merecen una lectura conceptual e histórica que busque puntos en común entre éstas diversas formas de expresión; por ello, la noción de campo de las pedagogías críticas pretende, pese a lo problemático que pueda resultar, darle un estatuto discursivo, pues práctico ya existe desde muchos escenarios como la educación popular, la educación comunitaria, prácticas de maestros en la educación formal, procesos gremiales y de movilización educativa, entre otros.

Diferenciaciones y acercamientos... El piso conceptual de la concomitancia entre campos.

Compartimos la lectura que propone Mario Díaz de la noción de campo, según la cual esta proviene del sociólogo Pierre Bourdieu y del filósofo Michel Foucault y fue empleada posteriormente por el sociolingüista Basil Bernstein para realizar una descripción metafórica de los diferentes contextos de la educación. El campo, así entendido, incluye tanto procesos estructurales como las formas de poder que surgen de los procesos de producción discursiva.

El campo de producción de discursos educativos sería, desde esta perspectiva, no un simple agregado de individuos creadores o fundadores de discursos, sino un dominio discursivopolítico que tendría efectos de control sobre la producción, distribución y circulación de su discurso. La producción del discurso educativo daría lugar a la generación de un campo dotado de una relativa autonomía social en los procesos discursivos mismos. Llamaremos a este campo, en congruencia con nuestros autores [Foucault, Bourdieu, Bernstein]2.

2 Díaz, Mario. (1993). El campo intelectual de la educación en CoIombia. Cali: Universidad del Valle, p. 10. 
Para Pierre Bourdieu, el campo ${ }^{3}$ es un principio de agrupación que permite analizar dentro del espacio social su división desde diferentes perspectivas: culturales, intelectuales, económicas y su relación directa con el poder. Un ejemplo específico al respecto lo da al referirse al campo científico ${ }^{4}$, el cual se puede entender cuando se observa el juego de fuerzas conceptuales en su interior, en términos de qué es la ciencia, qué es el conocimiento, cuáles son los procesos de objetivización de la realidad y cuáles son sus límites, la tensión entre lo objetivo y lo subjetivo en la construcción de conocimiento; así como las relaciones de validez y confiabilidad de los procedimientos y las acciones que puede llevar a cabo el investigador en este campo, además de observar las relaciones de poder que cruzan la producción de conocimiento y las relaciones entre los investigadores y con su objeto de investigación. Es entonces, siguiendo a Bourdieu, un campo de movimientos que se da entre lo que denomina el campo de producción cultural a gran escala (CGP) y el campo de la producción restringida $(\mathrm{CPR})^{5}$.

3 Un campo es del mismo modo que un campo magnético, un sistema estructurado de fuerzas objetivas, capaz de imponerse a todos los objetos y agentes que penetran en él. Pero a la vez, un campo es un espacio de conflictos y competición, en analogía con un campo de batalla en el que los contendientes rivalizan por establecer un monopolio sobre el tipo específico de capital eficiente en él: la autoridad cultural en el campo artístico, la autoridad científica en el campo científico, la autoridad sacerdotal en el campo religioso, etc.

4 El campo científico es un campo producto de las relaciones y estrategias de otros campos con los cuales interactúa y a los cuales recompone constantemente. En términos de Bourdieu, se trata de la acumulación del capital científico -cultural y simbólico- (prestigios, poder, beneficios, autoridad, competencia y reconocimiento). Bourdieu, Pierre. (1983). Campo intelectual, campo de poder y hábito de clase. En Campo del poder y campo intelectual. Buenos Aires: Folios Ediciones.

5 Esta división la plantea en el campo de la producción cultural-como mercado de bienes simbólicos. En el CPR, la ganancia económica es secundaria al realce del valor simbólico del producto y a la acumulación (a largo plazo) y gestación del capital simbólico, tanto por productores como por consumidores. Es un campo cerrado en sí mismo, con alto grado de autonomía; tiene poder para desarrollar sus propios criterios de producción y de evaluación y sus destinatarios son también productores por lo tanto, competidores. En el CGP hay un sometimiento a demandas externas: el mercado, la obra como mercancía. Su producción no puede ser incluida en la escala de los valores simbólicos, sus productos son de corta vida ya que son manejados como bienes económicos comunes y están destinados a un gran número de consumidores (el "gran público").
Es un campo de fuerzas en juego, fuerzas conceptuales, filosóficas, procedimentales, formas, figuras y relaciones imbuidos en una investigación, que en el caso de las pedagogías críticas nos permitiría, siguiendo a Bourdieu, cartografiar los mapas que se constituyen desde diferentes perspectivas pedagógicas y políticas, esos nuevos mapas desde donde se puedan reconocer espacios -territorios, límites, fronteras, puntos culminantes, topografías, accidentes, estructura del campo, acciones y estrategias-, buscando trazar los movimientos de las fuerzas existentes dentro de él.

Para Foucault, el campo es más bien una metáfora espacial donde se encuentran ubicadas las nociones de desplazamiento, posición y lugar; por el otro, están las metáforas geográficas: territorio, dominio, suelo, horizonte, archipiélago, región y paisaje. Las nociones de campo, posición, región, territorio, son formas de dominación que permiten la descripción de las relaciones de poder que atraviesan el saber6, así abre el camino para entender el proceso mediante el cual el saber funciona como un poder y reconduce a él los efectos y propone descifrar las transformaciones del discurso a través de metáforas espaciales, estratégicas, permitiendo captar con precisión los puntos en los cuales los discursos se transforman en, a través de y a partir de las relaciones de poder.

De otra parte, encontramos de acuerdo al mapa de concomitancia que hemos descrito, la propuesta del Campo Conceptual de la Pedagogía, elaborada por Olga Lucía Zuluaga y Alberto Echeverri, la cual surge en la búsqueda de reconocerle al campo pedagógico (CP) la posibilidad de reconceptualizar ${ }^{7} \mathrm{al}$ lado de la de recontextualizar, propuesta por el CIE de Mario Díaz.

6 Foucault, Michel (1992). Preguntas a Michel Foucault sobre la geografía. En Microfísica del Poder. Madrid: Ediciones de la Piqueta. $3^{\text {a }}$ edición, p. 116.

7 Para ubicar esta discusión: Zuluaga, Olga y Echeverri, Alberto. (2003). Campo intelectual de la educación y campo pedagógico: posibilidades, complementos y diferencias. En Pedagogía y Epistemología. Bogotá: Cooperativa Editorial Magisterio, pp. 111-125. 
El objetivo esencial de la configuración de un campo conceptual de la pedagogía sería buscar la comprensión del nuevo estatuto del saber y del campo pedagógico mismo, y aunque es descrito como un campo abierto, nos apartamos desde las pedagogías críticas, en el sentido de que aunque da prelación y centralidad a la pedagogía evitando subordinarla, no se observan con claridad las conexiones discursivas y prácticas con el análisis de contexto y con la condición social real de los sujetos maestros.

La diferencia fundamental con el campo de las pedagogías críticas está en la comprensión de la pedagogía no sólo como un campo conceptual autónomo y productor de conocimiento, aspectos que compartimos con el CP, sino como un campo político, es decir, desde nuestra lectura la pedagogía no es neutra, pero tampoco está subordinada y supeditada per se a las condiciones económicas, políticas y sociales de nuestro país, más bien reconoce la potencia política y biopolítica de los maestros desde la potenciación de la pedagogía como práctica política con implicaciones específicas en contextos particulares, y no con un impacto imaginario y etéreo.

El campo de las pedagogías críticas (CPC) en nuestro país se reconoce como un campo de concomitancia con el CP, pues la pedagogía y el maestro son el centro de los análisis; sin embargo, el CPC intenta reconocer las acciones desde una apuesta más local, concreta y real, tratando de incidir en las condiciones y actuaciones de los maestros e intentando producir cambios pedagógicos y políticos. Valoramos en el CP la potencia discursiva y la propuesta de articulación de diferentes puntos de vista que trabajan en torno a lo pedagógico (perspectiva hermenéutica, acción comunicativa, arqueología, genealogía, entre otros), y el análisis histórico que de manera aplicada se realiza en la propuesta conceptual de este campo en nuestro país.

Es así como la intencionalidad de esta propuesta está dirigida a tender puentes entre los discursos y prácticas que versan sobre la pedagogía y el maestro (en ocasiones un maestro y una pedagogía ideales) y realidades concretas y prácticas específicas. En ese sentido, consideramos que desde lecturas más políticas y que posibiliten la transformación del maestro, experiencias históricas de transformación propuestas por la Educación Popular8, el Movimiento Pedagógico de los maestros colombianos, algunas experiencias de la Expedición Pedagógica Nacional y de las redes de maestros, movilizaciones en defensa de la educación, algunas experiencias alternativas -con precauciones y distanciamientos hacia la noción misma de "innovación"-, habrían venido constituyendo y haciendo parte del campo de las pedagogías críticas en nuestro país, reiterando los procesos de concomitancia, pero también de diferenciación con las otras perspectivas teóricas de lectura que hemos mencionado anteriormente.

\section{Pedagogías críticas como discursos y prácticas de resistencia ${ }^{9}$}

No te salves

no te llenes de calma no reserves del mundo sólo un rincón tranquilo no dejes caer los párpados pesados como juicios no te quedes sin labios no te duermas sin sueño no te pienses sin sangre no te juzgues sin tiempo.

Mario Benedetti.

8 A la educación popular se le reconoce como una de las vías de apropiación de las pedagogías críticas en Colombia; en esa medida, las pedagogías críticas recogen los planteamientos de Paulo Freire desde la década de los ochenta en la educación no formal, configurando un enfoque que orienta los discursos, las prácticas y proyectos de nuestros contextos. Por ello asuntos como la relación práctica-teoría-práctica, es una construcción y confrontación permanente en cada uno de los escenarios en los que se actúa. Al respecto se identifican las producciones teóricas de investigadores y pedagogos a nivel nacional como Marco Raúl Mejía, Lola Cendales, Germán Mariño, Jorge Posada, Alfredo Ghiso, Alfonso Torres, Mario Acevedo, Mario Peresson, entre otros.

9 Proponemos la resistencia como una expresión de indignación, afirmación y compromiso que posibilita la construcción del vínculo social desde las diferencias y desigualdades. En esa medida también la asumimos como una praxis que moviliza sentidos de pertenencia y construcción de colectivos en pro de la justicia social en cada uno de sus órdenes. La resistencia se construye y se moviliza desde la memoria, el territorio, el cuerpo y las sensibilidades. 
La educación popular es una de las expresiones de las pedagogías críticas en Colombia reconocida como una corriente de pensamiento. En sus búsquedas por refundamentarse ha sido asumida desde procesos de escolarización y de desarrollo comunitario. En esta perspectiva han tenido lugar sus reflexiones desde las relaciones entre sujetos, política, pedagogía y proyecto político-cultural, configurando nuevos campos teóricos que, de alguna manera, responden a las tendencias actuales ubicadas en prácticas de animación socio-cultural y pedagogía social.

Sugiere Marco Raúl Mejía10, que desde la perspectiva de la educación popular, la pedagogía nos habla de una praxis educativa que es recontextualizada por actores con horizontes culturales diversos, siempre en lucha por el empoderamiento de los excluidos; asimismo, la educación popular nos ubica en una praxis humana que ubica en un plano dialógico relaciones sociales en permanente interlocución y conectando la vida social y cultural con los procesos educativos.

De acuerdo con los anteriores planteamientos, las pedagogías críticas en Colombia se están instituyendo como un campo de resignificación en torno a los modos de constitución de sujetos y al agenciamiento de procesos de formación desde una perspectiva política, ética y cultural. Las pedagogías son entendidas en esa medida como parte de un proyecto político-cultural, en el que la acción pedagógica se propone como lo plantea Joan Carles Mélich ${ }^{11}$ : como una relación de alteridad, una relación con el otro, una relación ética basada en la responsabilidad y hospitalidad. Por ello, se asume la escuela como un espacio social de formación, en contextos de diferencia y desigualdad.

Desde esta consideración, la pedagogía crítica se propone como un campo de crítica cultural, que problematiza e interroga las relaciones múltiples de subordinación. También como un discurso contex-

10 Mejía, Marco Raúl y Awad, Myriam Inés. (2003). Educación popular hoy. En tiempos de globalización. Bogotá: Ediciones Aurora, p. 83.

11 Mélich, Joan-Carles. (1997). Del extraño al cómplice. La educación en la vida cotidiana. Barcelona: Editorial Anthropos. tual, inserto en prácticas políticas concretas, que ha desafiado límites disciplinarios específicos tales como los estudios literarios, el feminismo, la antropología, la sociología y la psicología. Se inscribe en un lenguaje de esperanza y diálogo, comprometida críticamente. Giroux asume la pedagogía como

Una configuración de prácticas textuales, verbales y visuales que pretenden abordar los procesos mediante los cuales la gente se entiende a sí misma y comprende los modos en que afronta a los demás y su entorno. Reconoce que las presentaciones simbólicas que tienen lugar en diversas esferas de la producción cultural dentro de la sociedad ponen de manifiesto relaciones de poder cuestionadas y desiguales ${ }^{12}$.

Algunas claves para entender los elementos que conforman su campo de fundamentación ética, política y pedagógica están situadas en categorías fundantes y articuladoras como: sujeto, poder, conocimiento, cultura, historia, subjetividad, alteridad y contexto, que articulan procesos organizativos, formativos y de movilización social que responden a las tendencias y demandas actuales.

En esa medida se reconfiguran de manera permanente desde los escenarios escolares y no escolares, a partir de una lectura de contextos para poder aportar herramientas teóricas, diseñar estrategias políticas y proponer prácticas pedagógicas para el compromiso crítico y la transformación social en torno a procesos de constitución de sujetos y al agenciamiento de dinámicas culturales.

Se parte del presupuesto de concebir lo pedagógico como un espacio cultural para la construcción del conocimiento y la dinamización de espacios de socialización, lo que implica el reconocimiento de sujetos desde unos contextos que son diversos, desiguales y plurales. De ahí que la pedagogía haya dejado de ser exclusivamente escolar y ya no cumpla funciones sólo adaptativas y educativas, lo cual le lleva a interrogarse por su nuevo estatuto social orientado a la construcción del vínculo social, la

12 Giroux, Henry. (1997). Cruzando límites. Trabajadores culturales y políticas educativas. Barcelona: Paidós, p.16. 
tramitación de conflictos en múltiples escenarios sociales y la animación de procesos formativos, culturales y comunitarios.

La pedagogía crítica -en palabras de Giroux-es sobre todo una praxis política y ética, así como una construcción condicionada social e históricamente; la pedagogía no debe limitar su campo de acción a las aulas, sino que está comprometida con todas aquellas tentativas que pretenden influir en la producción y construcción de significado En este sentido, no tiene que ver únicamente con las prácticas de enseñanza, sino que implica también un reconocimiento de las políticas culturales que sustentan dichas prácticas. McLaren plantea que la pedagogía crítica es "esencialmente una pedagogía híbrida; es naturalmente anfibia. Está acostumbrada a disentir con climas intelectuales no llamados aún a un campo disciplinario propio. La pedagogía está enraizada éticamente, además de estar fundamentada teóricamente"13.

La pedagogía crítica es esencialmente una política de vida en la cual maestros y estudiantes están comprometidos en el trabajo de la historia; como tal ésta es una pedagogía que media con las relaciones concretas entre los individuos y la cultura y las formas institucionales, la cual lleva un compromiso social ${ }^{14}$. Por ello es una filosofía de la praxis, comprometida en un diálogo abierto en competencia con concepciones sobre cómo vivir significativamente en un mundo confrontado por la pena, el sufrimiento y la injusticia. La pregunta por la exclusión, la segregación, la marginación, la desigualdad y la represión en la esfera de lo social tienen formas educativas específicas y diferenciadas a través de una educación en la lucha y para la lucha. Es decir, se lee la realidad para transformarla, no simplemente para aprehenderla.

Giroux propone asumir la pedagogía crítica desde un enfoque de pedagogía de frontera o pedagogía de los límites, en la que se esté atento a desarrollar una filosofía pública democrática que respete la no-

13 Giroux, Henry y McLaren, Peter. (1998). Sociedad, cultura y educación. Madrid: Miño y Dávila Editores-Instituto Paulo Freire, p.226

14 Óp. cit, p. 225. ción de diferencia como parte de una lucha común por extender la calidad de la vida pública. Presupone no simplemente un reconocimiento de las fronteras cambiantes que socavan y también territorializan de nuevo diferentes configuraciones de la cultura, el poder y el conocimiento; también conecta las nociones de escolarización con una lucha más genuina por una sociedad democrática radical.

(....) La categoría de frontera denota un reconocimiento de esos márgenes epistemológicos, políticos, culturales y sociales que estructuran el lenguaje de la historia, el poder y la diferencia.

La categoría de frontera prefigura además la crítica cultural y los procesos pedagógicos como una forma de cruce de fronteras. Esto, es denota formas de trasgresión con que las fronteras existentes, forjadas desde la dominación, se pueden cuestionar y definir de nuevo.

En segundo lugar, también habla de la necesidad de crear las condiciones pedagógicas en que los estudiantes se conviertan en cruzadores de fronteras a fin de entender la alteridad en sus propios términos, y además crear zonas fronterizas donde los diversos recursos culturales permitan la confección de nuevas identidades dentro de las configuraciones existentes de poder ${ }^{15}$.

Uno de los sentidos vitales de las pedagogías críticas es la comprensión del sujeto, esto implica elaborar una política de la diferencia que se oponga activamente a la desvalorización de aquellos a quienes hemos relegado a la condición de "otro".

Deberíamos ser capaces de respetar la especificidad de las diferencias, articulando, al mismo tiempo, "diferencia" dentro de una política de solidaridad y de liberación en la que se generen y se realicen sueños mediante el desarrollo de comunidades de confianza y de afirmación, la pedagogía crítica es una pedagogía en la que lo personal es entendido siempre como social, y lo social es insertado siempre en la historia a fin de poner de manifiesto el modo particular en que ha sido producida la subjetividad, y elegir los caminos por medio de intereses de sexo, raza y clase ${ }^{16}$.

15 Óp. cit., p. 45

16 McLaren, Peter. (1994). Pedagogía crítica, resistencia cultural y la 
El discurso de muchos de los enfoques dominantes en el terreno de la pedagogía, se constituye recursivamente a través de visiones culturales ficticias del marginado, del que se aparta de la norma, del pobre, del desocupado y del de clase baja ${ }^{17}$, visiones que promueven la conformidad, la impotencia y la resignación.

Este nuevo discurso reconoce y hace suyas un conjunto de necesidades educativas (Giroux, 1998)18 como lo serían: 1) cómo discutir y emplear la comprensión de la diferencia para cambiar las relaciones prevalecientes de poder que la ubican en una situación de exclusión, 2) cómo analizar la colonización de la diferencia efectuada por los grupos dominantes, y cómo ésta es expresada y sostenida mediante representaciones en la que los otros son vistos como una deficiencia, 3) cómo descifrar críticamente la forma en que las voces de los otros son colonizadas y reprimidas por el principio de identidad que fluye a lo largo del discurso de los grupos dominantes y sobre todo 4) cómo crear nuevos espacios de discurso para reescribir narraciones culturales y definir los términos desde otra perspectiva, la de la otra parte.

Las PCs se asumen entonces como una instancia de producción cultural, política y ética, implicada en las formas de producción de subjetividades, en los procesos de construcción y circulación de valores y generación de prácticas sociales. Desde esta perspectiva, comunidades, organizaciones sociales e instituciones le apuestan a trabajar desde las pedagogías críticas como desencadenante de procesos socioeducativos, que nos permitan mayores y mejores cuotas de autonomía, participación, el respeto por la alteridad, espacios para la tramitación de los conflictos de manera creativa y la creación de ambientes sociales y comunitarios para reconocernos en nuestras apuestas colectivas.

producción del deseo. Instituto de estudios y acción social. Buenos Aires: Aique grupo editor, p.39

17 Óp. cit., p. 41.

18 Citado por Barraza, Arturo. La democracia y el discurso de la diferencia cultural: La pedagogía de frontera. En http:// www. Contexto- educativo.com.ar.

\section{Las pedagogías críticas ¿Por qué políticas y por qué éticas?}

\author{
Pero si \\ pese a todo \\ no puedes evitarlo \\ y congelas el júbilo \\ y quieres con desgana \\ y te salvas ahora \\ y te llenas de calma \\ y reservas del mundo \\ sólo un rincón tranquilo \\ y dejas caer los párpados \\ pesados como juicios \\ $\mathrm{y}$ te secas sin labios \\ $y$ te duermes sin sueño \\ $y$ te piensas sin sangre \\ y te juzgas sin tiempo \\ y te quedas inmóvil \\ al borde del camino \\ y te salvas \\ entonces \\ no te quedes conmigo
}

Mario Benedetti.

El discurso y la práctica misma de las pedagogías críticas no puede abstraerse del lenguaje de la diferencia y del poder, de allí que no pueda ser abordada solamente como una cuestión epistemológica o de procedimientos, sino que debe ser fundamentalmente definida en términos políticos y éticos.

Las pedagogías críticas son políticas dado que las formas y modos de "leer"la pedagogía y la educación contextualmente están vinculados ineluctablemente con relación al poder. De igual forma, son éticas desde la perspectiva de lectura de los sujetos (en este caso los maestros), lectura de sus prácticas, de los discursos educativos y pedagógicos, de los procesos de formación ciudadana y de los mismos contextos políticos locales donde desarrollan su quehacer cotidiano. Siguiendo a Giroux:"para los educadores críticos esto implica hablar acerca de importantes cuestiones sociales, políticas y culturales desde un profundo sentimiento de la política de su propio lugar" 19.

19 Giroux, Henry y Flecha, Ramón. (1994). Igualdad educativa y diferencia cultural. Barcelona: paidós, p. 14. 
En los contextos en los que se sitúa la pedagogía crítica reconoce problemáticas como el aumento de poblaciones en condiciones de desplazamiento, y asume una mirada crítica y a la vez propositiva frente a proyectos como "volver a la escuela" que atiende estudiantes en extra-edad, el aumento de estudiantes en preescolar y básica primaria con necesidades educativas especiales, niños, jóvenes y adultos en reinserción, procesos de alfabetización de adultos, poblaciones indígenas urbanizadas, desadaptación social, explotación sexual, diferentes formas de expresión de la violencia, la urgencia por atender una formación para el trabajo y las insatisfacciones existenciales.

Otro referente de análisis de las pedagogías críticas lo conforman las múltiples configuraciones de la cultura ${ }^{20}$. Estas se asumen desde la tradición y simultáneamente se reconfigura a partir de las nuevas narrativas de esta época. Las expresiones culturales son inestables, dialécticas, conflictivas y ponen en evidencia la creación de novedosas grupalidades, la conexión entre cultura, poder y saber, la semiotización de la vida cotidiana, la ausencia de proyectos a largo plazo y los nuevos procesos en la constitución de subjetividades. La pedagogía crítica trata sobre la problematización del lenguaje, de la experiencia, del poder, del conocimiento y de la cultura, cómo ellos son mutuamente constitutivos de la subjetividad y cómo sus conflictos generan una forma particular de praxis que actúa en y sobre el mundo.

El reconocimiento de la práctica del maestro como una práctica política pasa a su vez por el reconocimiento e interrogación de sí mismo como sujeto ético y político. Sin embargo, este reconocimiento se inserta de manera contextual con la problematización de la subalternidad y de la colonialidad social más general, de ahí que, siguiendo a Arturo Escobar, sea necesario un nuevo enfoque de la teoría

20 Esta puede leerse como clave estructurante en la construcción de sentidos. Creencias, códigos y símbolos que permiten a los sujetos interactuar, reconocerse y diferenciarse. Sistemas de significación que desarrolla modos de pensar, sentir y actuar construidos en los espacios de socialización. Cultura como performatividad y memoria. Tejido de expresiones en torno a la vida y a la existencia donde se construyen sentidos y significados que recrean la condición de los sujetos desde sus discursos, sensibilidades y actuaciones. social y éste quizás puede ser el "postmodernismo oposicional”. Así siguiendo a Escobar, quien cita a Boaventura de Sousa Santos:

Las condiciones que trajeron la crisis de la modernidad no son todavía las condiciones para superar la crisis más allá de la modernidad. De ahí la complejidad de nuestro período de transición relatado por la teoría oposicional postmoderna: estamos enfrentando problemas modernos para los cuales no hay soluciones modernas. La búsqueda de una solución es lo que denomino postmodernismo oposicional (...) Es necesario comenzar desde la disyunción de los problemas y la postmodernidad de las posibles soluciones, y convertir tales disyunciones en el impulso para fundamentar teorías y prácticas capaces de reinventar la emancipación social fuera de las destruidas promesas de emancipación de modernidad.

Escobar (2004,p.89) propone la necesidad de que la perspectiva latinoamericana de la modernidad/ colonialidad sugiera que las prácticas transformativas están produciéndose ahora y necesitan ser amplificadas socialmente. De allí que uno de los nichos políticos de las pedagogías críticas en nuestro país sea el reconocimiento tanto de las relaciones de poder que implica el sistema actual económico y educativo, como las relaciones de poder que son necesarias para una emancipación social, política y cultural. Lo anterior pasa por la necesidad de establecer no sólo discursos sino prácticas de oposición -siguiendo nuevamente a Escobar- educativas y pedagógicas frente a la exclusión, a los procesos de regulación educativos y al control mismo de su práctica pedagógico-política, poniendo en tela de juicio el actual marco de aceptación de las desigualdades sociales.

Asimismo, en el marco de profundas transformaciones del sistema educativo en nuestro país orientadas por políticas internacionales, es prioritario cuestionarse sobre el lugar que ocupa la dimensión ético-política de propuestas alternativas como las que se agrupan en el campo de las pedagogías críticas en Colombia con los usos instrumentales y homogenizantes de la reforma educativa, los cuales actúan como dispositivos de adaptación y abren 
la posibilidad de discusión sobre las relaciones de la justicia con las políticas públicas en educación. Cullen (1999) nos presenta los que él denomina modelos disfuncionales de focalización de las políticas educativas a saber:

a) la variable cultural de la integración-homogenizante (bajo el predominio del valor 'pertenencia libre' relacionado con la formación de nuevas identidades sociales modernas), y entonces un Estado neutral, que permite incluir las diferencias en la formación de una nación construida desde un pacto social.b) la variable económica de desarrollo de capital humano (bajo el predominio de igualdad de oportunidades), y entonces un Estado posibilitador que permite movilizar las diferencias de acuerdo con el esfuerzo de cada uno. c) la variable social de la compensación de las desigualdades (bajo el valor -integrador- de la equidad) y entonces un Estado benefactor, que permite solamente las desigualdades que maximizan las ventajas de los menos favorecidos ${ }^{21}$.

Estos tres modelos, como lo plantea Cullen (1999), en su constante intervención e interrelación en las políticas públicas educativas potencializan el valor estratégico de la compensación de las desigualdades sociales.

Sin embargo, a pesar de la tendencia homogenizante del sistema económico y político mundial, se dinamizan propuestas de resistencia como la del Foro Temático Mundial de Porto Alegre: "Otro mundo es posible”, en el pensamiento más allá del Tercer Mundo, "está en juego la habilidad de imaginar tanto 'otros mundos' como 'mundos de otro modo' esto es, mundos que son más justos y sustentables y, al mismo tiempo, mundos que son definidos mediante otros principios antes que los de la modernidad eurocentrada"22. Y, siguiendo a Escobar, nos podemos cuestionar sobre: ¿Cuáles son los sitios de donde provienen las ideas de estas imaginaciones alternativas y disidentes para el caso Colombiano? ¿Cómo son las imaginaciones disidentes puestas en

21 Óp. cit., pp. 85-86.

22 Ibíd. movimiento? ¿Se restringen únicamente a contextos no escolares? ¿Cuáles son las reales posibilidades de emergencia en contextos escolares formales? ¿Existen tanto desde las movilizaciones sociales como desde la práctica pedagógica alternativa y crítica de algunos maestros? ¿Son reales o ideales?

Consideramos entonces a partir de estos planteamientos, que las pedagogías críticas en nuestro país agrupan prácticas, experiencias y movilizaciones que buscan responder a los cuestionamientos anteriormente expuestos, desde el abordaje de contextos particulares de acción, con la aclaración de que identificar que somos diferentes, no implica asumir de manera esencialista nuestro devenir histórico, por el contrario, apunta al reconocimiento del marco de desigualdad social existente en donde emergen y se producen diferencias culturales y políticas que no se pueden obviar ni tampoco invisibilizar para el ámbito educativo de manera específica. En este marco, las pedagogías críticas podrían releer las acciones que históricamente ha venido realizando en la posibilidad de construcción de políticas de la diferencia ${ }^{23}$, que potencien las acciones del sujeto maestro como sujeto político.

Un asunto de debate en la formulación de políticas educativas es problematizar las concepciones de sujetos sobre los cuales éstas se diseñan, como: "pobres", "necesitados", "en alto riesgo", "en vulnerabilidad”, "marginales", “minorías”, “minusválidos”, "discapacitados”. Las pedagogías críticas proponen deconstruir estas concepciones y resignificarlas como sujetos de derechos en condiciones de diferencia y desigualdad social, asimismo deconstruir los imaginarios homogeneizantes y de control que sostienen estas concepciones. Las pedagogías críticas son en este sentido "esencialmente una política de vida en la cual profesores y estudiantes

23 Más que de reconocimiento de la diversidad, pues el uso y funcionamiento de la noción de diversidad en las políticas públicas educativas está en estudio y profundización al interior del grupo de investigación, como una noción cooptada y articulada a la de población vulnerable donde se involucra tanto a campesinos, como a desplazados, sujetos de etnias distintas, población indígena, homosexuales, entre otros, sin diferenciación alguna y sin tener en cuenta el contexto de desigualdad social en el que realmente funcionan las llamadas políticas de inclusión. 
están comprometidos en el trabajo de la historia; como tal esta es una pedagogía que media entre con las relaciones concretas entre los individuos y la cultura y las formas institucionales, la cual lleva un compromiso social“24.

Las pedagogías críticas comparten con la educación popular una apuesta ética y política, sustentada por fines de reconocimiento, justicia social, empoderamiento y democracia de sujetos que se encuentran desde sus diferencias y desigualdades en condiciones de género, clase, etnia, sexo y condiciones de subalternidad; una actuación intencionada de corte educativo que construye lo colectivo como soporte de los procesos escolares y sociales, una apuesta pedagógica en la orientación de procesos de construcción del conocimiento y de socialización que incluye prácticas, saberes, dinámicas socioculturales e interacciones para transformar la sociedad desde las resistencias culturales.

Consideramos finalmente que las pedagogías críticas proponen a maestros de universidades, de escuela básica y media, a líderes comunitarios y agentes culturales el desarrollo de una sensibilidad crítica y de no indiferencia que sea extensión de su conciencia histórica. Esto es, reconocer que la realidad no es natural ni estática, sino que es algo en cuya construcción participamos y cuyas lógicas deben cuestionarse y analizarse continuamente. Desde una intencionalidad de trabajo educativo cultural se justifica entonces que se generen dinámicas de vinculación donde se construyan significados de vida para maestros, estudiantes y actores comunitarios y que asimismo estas dinámicas sean escenarios para la constitución de identidades colectivas que hagan posible la apropiación de espacios, el sentido de pertenencia, la construcción de nichos afectivos y la configuración de nuevas subjetividades.

Apostar desde las pedagogías críticas a la construcción de vínculos significa reconfigurar las relaciones sociales que posibiliten el reconocimiento de las identidades fluctuantes, fronterizas, de estudiantes y maestros, legitimar sistemas de regulación social construidos colectivamente, abrir las puertas de la escuela a la vida, generando espacios donde realmente se aprenda a vivir con los otros, espacios donde circulen las narrativas construidas por cada uno de los actores educativos, narrativas que permitan ver la singularidad de éstos. Sin singularidades no es posible la diferencia.

La existencia humana en tanto humana no puede ser muda, silenciosa, ni tampoco nutrirse de falsas palabras, sino de palabras verdaderas con las cuales los hombres transforman el mundo. Los hombres no se hacen en el silencio, sino en la palabra. Decirla no es privilegio de algunos sino derecho de todos los hombres. El diálogo es este encuentro de los hombres, mediatizados por el mundo, para pronunciarlo25. 凤

Paulo Freire
24 En Giroux, Henry y McLaren, Peter. (1998). Sociedad, cultura y educación. Madrid: Miñó y Dávila Editores-Instituto Paulo Freire, p. 225.
25 Freire, Paulo. (1997). Educación en la ciudad. México. Siglo XXI Editores. 


\section{Bibliografía}

Barcena, Fernando y MÉLICH, Joan-Carles. (2000). La educación como acontecimiento ético. Papeles de pedagogía. Barcelona: Paidós.

Cullen, Carlos. (1999). Autonomía moral, participación democrática y cuidado del otro. Bases para un currículum de formación ética y ciudadana. Buenos Aires: Ediciones Novedades Educativas. 2a ${ }^{\mathrm{a}}$. Edición.

Díaz, Mario. (1993). El campo intelectual de la educación en Colombia. Cali: Universidad del Valle.

Escobar, Arturo. (2004). Más allá del Tercer Mundo: Globalidad imperial, colonialidad global y movimientos sociales antiglobalización. Revista Nómadas, 20, Abril.

Foucault, Michel. (1992). Preguntas a Michel Foucault sobre la geografía. En Microfísica del Poder. Madrid: Ediciones de la Piqueta. $3^{a}$. edición.

Franco, Yago. (2000). Subjetividad: lo que le mercado se llevó: una perspectiva desde l pensamiento de Cornelius Castoriadis. Recuperado de http://www.magma-net. com.ar/subjetividad.htm. Consultado el 12 de junio de 2007.

Freire, Paulo. (1970). Pedagogía del oprimido. Buenos Aires: Siglo XXI Editores.

Freire, Paulo. (2006). Pedagogía de la autonomía. México: Siglo XXI Editores.

Giroux, Henry. (1992). Teoría y resistencia en educación. México: Siglo XXI Editores.

Giroux, Henry y Flecha, Ramón. (1994). Igualdad educativa y diferencia cultural. Barcelona: Paidós.

Giroux, Henry. (1997). Cruzando límites. Trabajadores culturales y políticas educativas. Barcelona:Paidós.

Giroux, Henry y Aclaren, Peter. (1998). Sociedad, cultura y educación. Madrid: Miño y Dávila Editores-Instituto Paulo Freire.
Larrosa, Jorge y Skliar, Carlos. (2001). Habitantes de babel. Políticas y poéticas de la diferencia. Barcelona: Laertes.

Martínez Boom, Alberto. (1998). Crónicas del desarraigo. Historia del maestro en Colombia. Revista Educación y Cultura, 16.

McLaren, Meter. (1994). Pedagogía crítica, resistencia cultural y la producción del deseo. Argentina: Aique Grupo Editor.

Mejía, Marco Raúl y Awad, Myriam Inés. (2003). Educación popular hoy. En tiempos de globalización. Bogotá: Ediciones Aurora.

Mejía, Marco Raúl. (2007), Educación (es) en la (s) globalización (es). I. Entre el pensamiento único y la nueva crítica. Bogotá: Ediciones desde abajo.

Mélich, Joan-Carles. (1994). Del extraño al cómplice. La educación en la vida cotidiana. Barcelona: Antrophos.

Zaccagnini, Mario. Configuraciones del sujeto pedagógico contemporáneo. Intersecciones; tensiones y fracturas; continuidades y discontinuidades, entre la epistemología de las prácticas educativas escolares y la realidad social. Grupo G.I.S.E.A. Universidad Nacional de Mar del Plata. Argentina. Recuperado de http://www.fchst.unlpam.edu.ar/iciels/171.pdf. Consultado el 18 de junio de 2007.

Zuluaga, Olga y Echeverri, Alberto. (2003). Campo intelectual de la educación y campo pedagógico: posibilidades, complementos y diferencias. En Pedagogía y Epistemología. Bogotá: Cooperativa Editorial Magisterio. 
